\title{
INVERSE SEMIGROUPS WITH ZERO: COVERS AND THEIR STRUCTURE
}

\author{
SYDNEY BULMAN-FLEMING, JOHN FOUNTAIN and VICTORIA GOULD
}

(Received 9 August 1997; revised 29 March 1999)

Communicated by D. Easdown

\begin{abstract}
We obtain analogues, in the setting of semigroups with zero, of McAlister's covering theorem and the structure theorems of McAlister, O'Carroll, and Margolis and Pin. The covers come from a class $C$ of semigroups defined by modifying one of the many characterisations of $E$-unitary inverse semigroups, namely, that an inverse semigroup is $E$-unitary if and only if it is an inverse image of an idempotent-pure homomorphism onto a group. The class $C$ is properly contained in the class of all $E^{*}$-unitary inverse semigroups introduced by Szendrei but properly contains the class of strongly categorical $E^{*}$-unitary semigroups recently considered by Gomes and Howie.
\end{abstract}

1991 Mathematics subject classification (Amer. Math. Soc.): primary $20 \mathrm{M} 18$.

Keywords and phrases: $P$-theorem, $E^{*}$-unitary, cover.

\section{Introduction}

We assume that the reader is familiar with the classical theory of $E$-unitary inverse semigroups due to McAlister [5, 6] as presented, for example, in [3]. The essence of this theory is that every inverse semigroup is 'closely related' to an $E$-unitary inverse semigroup (by having an $E$-unitary inverse cover), and that the structure of $E$-unitary inverse semigroups is well understood (by virtue of what is now known as the $P$-theorem). Another description of $E$-unitary inverse semigroups was given by O'Carroll [8] who characterised them as the inverse semigroups which can be embedded in a semidirect product of a semilattice and a group. In [4], Margolis and Pin introduced a different approach to the study of (not necessarily regular) $E$-unitary

The research, and the visit of the third author to Wilfrid Laurier University were supported by Natural Sciences and Engineering Research Council of Canada Grant A4494.

(C) 1999 Australian Mathematical Society 0263-6115/99 \$A2.00+0.00 
semigroups by making use of groups acting on categories. They were able to recover the $P$-theorem from their more general results.

When dealing with inverse semigroups with zero, the theory outlined above is unsatisfactory for the reason that an $E$-unitary inverse semigroup with zero is necessarily a semilattice. Consequently, the only inverse semigroups with zero which have an $E$-unitary cover with a zero are semilattices. Our aim in this paper is to obtain both a covering theorem for all inverse semigroups with zero and a structure theorem for the covers. The semigroups we use as covers belong to a class, which for the class of inverse semigroups with zero, is one analogue, among many, of the class of $E$-unitary inverse semigroups. There are many characterisations of $E$-unitary inverse semigroups and we now remind the reader of the one of particular interest for us. Recall that a function $\varphi: S \rightarrow T$ from a semigroup $S$ to a semigroup $T$ is idempotent-pure if $a$ is idempotent whenever $a \varphi$ is idempotent. An inverse semigroup $S$ is $E$-unitary if and only if there is an idempotent-pure homomorphism from $S$ onto a group. This is the condition which we adapt to the case of semigroups with zero.

A function $\varphi: S \rightarrow T$ between semigroups with zero is 0 -restricted if $0 \varphi^{-1}=\{0\}$.

DEFINITION 1. Let $S$ be a semigroup with zero and $G^{0}$ be a group with zero. A 0-restricted function $\varphi: S \rightarrow G^{0}$ is a 0-morphism if $(s t) \varphi=s \varphi t \varphi$ for all $s, t \in S$ with $s t \neq 0$.

DEFINITION 2. An inverse semigroup $S$ with zero is strongly $E^{*}$-unitary if there is an idempotent-pure 0 -morphism $\varphi$ from $S$ into a group with zero.

Let $T$ be a subset of a semigroup $S$ with zero. The set of non-zero elements of $T$ is denoted by $T^{*}$, and $E^{*}(S)$ denotes the set of non-zero idempotents of $S$.

An inverse semigroup $S$ is $E^{*}$-unitary [9] if $S$ has a zero and whenever $a \in S, e \in$ $E^{*}(S)$, and either $e a$ or $a e$ belongs to $E^{*}(S)$, then $a \in E^{*}(S)$. While it is true that every inverse semigroup with zero has an $E^{*}$-unitary inverse cover, there is, as yet, no structure theorem for general $E^{*}$-unitary inverse semigroups.

We observe that every strongly $E^{*}$-unitary inverse semigroup is $E^{*}$-unitary. The converse, however, is not true. In Section 1 we give an example of a commutative inverse semigroup with zero that is $E^{*}$-unitary but not strongly $E^{*}$-unitary.

Adapting the classical notion of cover, we say that a semigroup $P$ with zero is a 0 -cover of a semigroup $S$ with zero if there is a surjective homomorphism $\psi: P \rightarrow S$ that is 0 -restricted and idempotent-separating. We begin Section 1 by showing that every inverse semigroup with zero has a strongly $E^{*}$-unitary inverse 0 -cover. Next we discuss groups acting on semigroupoids and the way in which such actions give rise to strongly $E^{*}$-unitary inverse semigroups. We then introduce McAlister 0-triples to give another construction of such semigroups. 
The main result of Section 2 is a structure theorem for strongly $E^{*}$-unitary inverse semigroups which includes an analogue of McAlister's $\boldsymbol{P}$-theorem. It also contains two other characterisations of these semigroups corresponding to results of O'Carroll [8] and Margolis and Pin [4]. We show that they are precisely the inverse subsemigroups containing zero of 0 -semidirect products of semilattices with zero by groups, and that they can also be described in terms of groups acting on inverse semigroupoids.

In [2], Gomes and Howie obtain a type of $P$-theorem for strongly categorical $E^{*}$ unitary inverse semigroups. Munn [7] has shown that a strongly categorical inverse semigroup $S$ admits a 0 -restricted homomorphism onto a Brandt semigroup $S / \beta$ (its largest Brandt homomorphic image). It is shown in [2] that such a semigroup $S$ is $E^{*}$-unitary if and only if $\beta$ is idempotent-pure. We use this to show that if $S$ is an $E^{*}$-unitary inverse semigroup which is strongly categorical, then $S$ is strongly $E^{*}$-unitary.

In the final section we specialise to the case of $E^{*}$-unitary inverse semigroups that are strongly categorical. Using our results we recover the $P$-theorem of [2].

\section{Rees quotients of $E$-unitary inverse semigroups}

In this section we give our covering theorems and introduce two constructions of strongly $E^{*}$-unitary inverse semigroups. The first construction is based on a group acting on a semigroupoid and the second is our analogue of a $P$-semigroup. We adapt the theory of $E$-unitary inverse semigroups by using the following simple lemma.

\section{LEMMA 1.1. Every Rees quotient of an E-unitary inverse semigroup $S$ is strongly $E^{*}$-unitary.}

PROOF. Let $\varphi: S \rightarrow G$ be an idempotent-pure homomorphism from $S$ to a group $G$, and let $I$ be an ideal of $S$. Then $S / I$ is inverse, and $\bar{\varphi}: S / I \rightarrow G^{0}$ given by $s \bar{\varphi}=s \varphi$ for $s \in S \backslash I$ and $I \bar{\varphi}=0$ is an idempotent-pure 0 -morphism.

We now have our covering result.

PROPOSITION 1.2. Every inverse semigroup with zero has a strongly $E^{*}$-unitary inverse 0-cover.

ProOF. Let $S$ be an inverse semigroup with zero. Then $S$ has an $E$-unitary cover, that is, there is an $E$-unitary inverse semigroup $P$ and a surjective homomorphism $\psi: P \rightarrow S$ having the property that $\psi$ restricted to $E(P)$ is an isomorphism of $E(P)$ onto $E(S)$. 
Let $J$ denote the ideal $0 \psi^{-1}$ of $P$. Then clearly $\psi$ factors through $P / J$ via a surjective homomorphism $\eta: P / J \rightarrow S$ which is 0-restricted and idempotentseparating. By Lemma $1.1, P / J$ is strongly $E^{*}$-unitary.

We recall that semigroup $S$ is categorical at zero if $S$ has a zero and for all $a, b, c \in S$, if $a b \neq 0$ and $b c \neq 0$, then $a b c \neq 0$. An inverse semigroup $S$ is strongly categorical if $S$ is categorical at zero and, in addition, the intersection of any two non-zero ideals of $S$ is non-zero.

COROLLARY 1.3. Every strongly categorical inverse semigroup has an $E^{*}$-unitary inverse 0-cover which is strongly categorical.

ProOF. Let $S$ be a strongly categorical inverse semigroup. By Proposition 1.2, $S$ has a strongly $E^{*}$-unitary inverse 0 -cover $Q$. Let $\eta: Q \rightarrow S$ be a covering homomorphism. By [7, Theorem 2.7], there is a 0-restricted homomorphism $\lambda: S \rightarrow B$ onto a Brandt semigroup $B$. Hence $\eta \lambda$ is a 0 -restricted homomorphism from $Q$ onto $B$ and so by [7, Theorem 1.1], $Q$ is strongly categorical.

A semigroupoid $C$, also known as a 'category without identities', or 'quiver' in the terminology of [1], consists of a set of objects $\mathrm{Ob} C$ and for each ordered pair $(u, v)$ of objects a set of morphisms $\operatorname{Mor}(u, v)$ which may be empty. The set $\bigcup_{u, v \in \mathrm{Ob} C} \operatorname{Mor}(u, v)$ of all morphisms is denoted by Mor $C$ and there is a partial operation called composition on Mor $C$ which satisfies the axioms of a category except for those involving identities. Many common semigroup terms can be easily adapted to semigroupoids.

A semigroupoid $C$ is regular if for all objects $u, v \in \operatorname{Ob} C$ and for all $p \in \operatorname{Mor}(u, v)$, there exists $q \in \operatorname{Mor}(v, u)$ such that $p+q+p=p$, where here, as elsewhere, we use + to denote composition of morphisms. The morphism $q$ is an inverse of $p$ if, in addition, $q+p+q=q$. Notice that if $p+q+p=p$, then $q+p+q$ is an inverse of $p$. A semigroupoid is inverse if every morphism has a unique inverse.

For each object $u$ of a semigroupoid $C$, the set of morphisms $\operatorname{Mor}(u, u)$ is a semigroup (possibly empty) under the operation of composition. The semigroups $\operatorname{Mor}(u, u)$ are called the local semigroups of $C$ and if $P$ is a semigroup property, then $C$ is said to be locally $P$ if all its local semigroups have property $P$. We note that a regular semigroupoid is inverse if and only if it is locally inverse.

A non-empty subset $I$ of Mor $C$ is an ideal if, whenever $p \in I, q \in \operatorname{Mor} C$, and $p+q(q+p)$ is defined, then $p+q(q+p)$ is in $I$.

We remark that the definitions and properties of Green's relations carry over to semigroupoids in the obvious way.

A group $G$ acts on a semigroupoid $C$ (on the left) if $G$ acts on $\mathrm{Ob} C$ and on Mor $C$, and for all $g \in G$, if $p \in \operatorname{Mor}(u, v)$, then $g p \in \operatorname{Mor}(g u, g v)$ and if $q \in \operatorname{Mor}(v, w)$, 
then $g(p+q)=g p+g q$. The group $G$ acts transitively if, for any $u, v \in \mathrm{Ob} C$, there exists $g \in G$ with $g u=v$. It acts freely when for any $g \in G$ and $u \in \mathrm{Ob} C$, if $g u=u$, then $g=1$.

Now let $G$ be a group acting freely on a semigroupoid $C$. Fix an object $u$ in $\mathrm{Ob} C$ and let

$$
C_{u}=\{(p, g) \in \operatorname{Mor} C \times G: p \in \operatorname{Mor}(u, g u)\}
$$

and define a multiplication on $C_{u}$ by

$$
(p, g)(q, h)=(p+g q, g h) .
$$

Then $C_{u}$ is a semigroup.

Note that while [4] is concerned with groups acting on categories, the proofs of results relevant here are easily adapted to semigroupoids. The following proposition is contained in [4, Propositions 3.11 and 3.14]. We note that the part of the proof of Proposition 3.14 used for the inverse semigroupoid result does not require the semigroupoid to be connected. We observe further that when the action is transitive and free, $C_{u} \cong C_{v}$ for any objects $u, v$ of a semigroupoid $C$.

PROPOSITION 1.4. Let $G$ be a group acting transitively and freely on an inverse, locally idempotent semigroupoid $C$. Let $u \in \mathrm{Ob} C$. Then $C_{u}$ is E-unitary inverse.

PROPOSITION 1.5. Let $G$ be a group acting transitively and freely on an inverse, locally idempotent semigroupoid C. Suppose that $I$ is an ideal of $C$ and $G I \subseteq I$. Let $u \in \mathrm{Ob} C$ and put

$$
I_{u}=\left\{(p, g) \in C_{u}: p \in I\right\} .
$$

Then $I_{u}$ is an ideal of $C_{u}$ and the Rees quotient $C_{u} / I_{u}$ is strongly $E^{*}$-unitary.

Proof. Since $G$ acts transitively, we see that $I_{u}$ is non-empty, and clearly, $I_{u}$ is an ideal of $C_{u}$. The remainder of the proposition follows from Lemma 1.1 and Proposition 1.4.

In our main result, Theorem 2.5 , we show that all strongly $E^{*}$-unitary inverse semigroups can be obtained using the construction of Proposition 1.5. To this end we adapt the notion of the weak derived category of a monoid morphism as given, for example, in [4] where it is called the derived category.

Let $S$ be a strongly $E^{*}$-unitary inverse semigroup. Then there is a group $G$ and idempotent-pure 0-morphism $\varphi: S \rightarrow G^{0}$ from $S$ into $G^{0}$. We construct a semigroupoid $C$ from $S, G$ and $\varphi$ by defining

$$
\mathrm{Ob} C=G
$$


and for $a, b \in G$,

$$
\operatorname{Mor}_{C}(a, b)=\{(a, s, b): s \in S, s=0 \text { or } a(s \varphi)=b\}
$$

With composition of morphisms given by

$$
(a, s, b)+(b, t, c)=(a, s t, c)
$$

$C$ is a semigroupoid. The group $G$ acts on $C$ with the action on objects given by group multiplication and the action on morphisms given by $g(a, s, b)=(g a, s, g b)$. Clearly, $G$ acts transitively and freely on $C$. We now gather together some facts concerning $C$.

PROPOSITION 1.6. Let $S$ be a strongly $E^{*}$-unitary inverse semigroup, $G$ be a group, and $\varphi: S \rightarrow G^{0}$ be an idempotent-pure 0-morphism. Then, for $a, b, c, d \in G$ and $s, t \in S$,

(1) $C$ is an inverse semigroupoid where for all $(a, s, b) \in \operatorname{Mor} C$, the inverse of $(a, s, b)$ is $\left(b, s^{-1}, a\right)$;

(2) $\operatorname{Mor}(a, a)=\{(a, e, a): e \in E(S)\}$ is a semilattice isomorphic to $E(S)$, for every $a \in G$; in particular, $C$ is locally idempotent and locally commutative;

(3) if $(a, s, b) \mathscr{J}(a, t, b)$, then $s=t$;

(4) $\mathscr{J}=\mathscr{D}$ in $C$;

(5) $(a, s, b) \mathscr{R}(c, t, d)$ if and only if $a=c$ and $s \mathscr{R} t$, and $(a, s, b) \mathscr{L}(c, t, d)$ if and only if $b=d$ and $s \mathscr{L} t$;

(6) $(a, s, b) \mathscr{D}(c, t, d)$ if and only if $s=t=0$ or there exists $r \in S^{*}$ such that $a(r \varphi)=d, s s^{-1}=r r^{-1}$ and $r^{-1} r=t^{-1} t$.

ProOF. To show (1) is routine; (2) follows from the fact that $\varphi$ is idempotent-pure. For (3), simply note that [4, Proposition 3.4] holds for semigroupoids. The fact that $\mathscr{D}=\mathscr{J}$ follows from [4, Proposition 3.1]. The proof of (5) is routine and (6) follows easily.

We now modify the usual notion of $P$-semigroup so that we obtain strongly $E^{*}$ unitary inverse semigroups. This is, in fact, not difficult. Recall from [3, Section 5.9] that a triple $(G, \mathscr{X}, \mathscr{Y})$ is a McAlister triple if $G$ is a group which acts by order automorphisms on a partially ordered set $\mathscr{X}$, and $\mathscr{Y}$ is a subsemilattice of $\mathscr{X}$ (in the sense that for every $J, K \in \mathscr{Y}$, there is a greatest lower bound of $J$ and $K$, also in $\mathscr{Y}$ ) and an order ideal of $\mathscr{X}$ such that

(i) $G \mathscr{Y}=\mathscr{X}$, and

(ii) $g \mathscr{Y} \cap \mathscr{Y} \neq \emptyset$ for all $g \in G$. 
If in addition $\mathscr{X}$ has a smallest element 0 , we say that $(G, \mathscr{X}, \mathscr{Y})$ is a McAlister 0 -triple. Note that in this case, since $g 0=0$, the condition that $g \mathscr{Y} \cap \mathscr{Y} \neq \emptyset$ for all $g \in G$ is automatically satisfied.

Let $(G, \mathscr{X}, \mathscr{Y})$ be a McAlister triple. Then $\mathscr{M}(G, \mathscr{X}, \mathscr{Y})$ denotes the ' $P$ semigroup' with universe

$$
\left\{(A, g) \in \mathscr{Y} \times G: g^{-1} A \in \mathscr{Y}\right\}
$$

and multiplication defined by

$$
(A, g)(B, h)=(A \wedge g B, g h)
$$

From [5] and [6], the classes of $P$-semigroups and $E$-unitary inverse semigroups coincide.

LEMMA 1.7. If $(G, \mathscr{X}, \mathscr{Y})$ is a McAlister 0-triple, then $g 0=0$ for any $g \in G$, and $\{0\} \times G$ is the unique minimum ideal of $P=\mathscr{M}(G, \mathscr{X}, \mathscr{Y})$.

PROOF. The first statement is clear and it follows that $\{0\} \times G$ is an ideal of $P$. Let $J$ be an ideal of $P$ and let $(A, g) \in J$. If $h \in G$, then $\left(0, g^{-1} h\right) \in P$ and since $(0, h)=(A, g)\left(0, g^{-1} h\right)$ we see that $(0, h) \in J$. Thus $\{0\} \times G$ is the unique minimum ideal, as required.

Let $(G, \mathscr{X}, \mathscr{Y})$ be a McAlister 0-triple. Throughout the paper, $\mathscr{M}_{0}(G, \mathscr{X}, \mathscr{Y})$ denotes the Rees quotient of $\mathscr{M}(G, \mathscr{X}, \mathscr{Y})$ by its ideal $\{0\} \times G$. The following result is immediate from Lemma 1.1.

COROLLARY 1.8. With the above notation, $\mathscr{M}_{0}(G, \mathscr{X}, \mathscr{Y})$ is a strongly $E^{*}$-unitary inverse semigroup.

REMARK. When $\mathscr{X}=\mathscr{Y}$ the semigroup $\mathscr{M}(G, \mathscr{Y}, \mathscr{Y})$ is just a semidirect product $\mathscr{Y} * G$ of the semilattice $\mathscr{Y}$ and the group $G$. If $\mathscr{Y}$ has a zero, then $\mathscr{M}_{0}(G, \mathscr{Y}, \mathscr{Y})$ is the 0 -semidirect product introduced by Szendrei [9]. It is the Rees quotient of $\mathscr{Y} * G$ by the ideal $\{0\} \times \mathscr{Y}$ and is denoted by $\mathscr{Y} *_{0} G$. Noting that an inverse subsemigroup containing zero of a strongly $E^{*}$-unitary inverse semigroup is strongly $E^{*}$-unitary, the following corollary is immediate.

COROLLARY 1.9. Every inverse subsemigroup containing zero of a 0 -semidirect product of a semilattice and a group is strongly $E^{*}$-unitary.

In the next section, we will see that every strongly $E^{*}$-unitary inverse semigroup arises in the manner described in Proposition 1.5, Corollary 1.8 and Corollary 1.9. 
We conclude this section with an example of an $E^{*}$-unitary inverse semigroup which is not strongly $E^{*}$-unitary.

Clearly, a Clifford semigroup has a zero if and only if it is a semilattice $Y$ of groups $G_{\alpha}(\alpha \in Y)$ where $Y$ has a least element 0 and $G_{0}$ is the trivial group. Further, such a semigroup is $E^{*}$-unitary if and only if for all $\alpha, \beta \in Y$ with $\alpha \geq \beta \neq 0$, the connecting homomorphism $\varphi_{\alpha, \beta}$ is one-to-one.

EXAMPLE. Let $Y$ be the free semilattice on three generators $\alpha, \beta, \gamma$; write 0 for the zero $\alpha \beta \gamma$ of $Y$. For each $\mu \in Y^{*}$, let $G_{\mu}$ be the infinite cyclic group with generator $a_{\mu}$ and identity $e_{\mu}$, and let $G_{0}=\{0\}$. For $\mu, \nu \in Y$ with $\mu \geq \nu \neq 0$, let $\varphi_{\mu, \nu}$ be the injective homomorphism induced by the rule:

$$
a_{\mu} \varphi_{\mu, v}= \begin{cases}a_{v}^{2} & \text { if } \mu=\alpha \text { and } v=\alpha \beta \\ a_{\nu} & \text { otherwise. }\end{cases}
$$

Let $S$ be the semilattice $Y$ of groups $G_{\alpha}(\alpha \in Y)$ constructed with these connecting homomorphisms (there is no choice for the ones remaining undefined). From the comments above, $S$ is $E^{*}$-unitary. However, $S$ is not strongly $E^{*}$-unitary, for if there were an idempotent-pure 0-morphism $\theta$ from $S$ to a group with zero, then since $a_{\mu} \varphi_{\mu, \nu}=a_{\mu} e_{v}$, we would have

$$
a_{\alpha \beta}^{2} \theta=a_{\alpha} \theta=a_{\alpha \gamma} \theta=a_{\gamma} \theta=a_{\beta \gamma} \theta=a_{\beta} \theta=a_{\alpha \beta} \theta,
$$

contradicting the fact that $a_{\alpha \beta}$ is not idempotent.

\section{A characterisation theorem}

Before stating and proving the main result of this section, Theorem 2.5 , we present some preliminary material to help clarify the exposition.

Let $S$ be a strongly $E^{*}$-unitary inverse semigroup. Then there is a group $G$ and an idempotent-pure 0 -morphism $\varphi: S \rightarrow G^{0}$. Let $C$ be the semigroupoid constructed from $S, G$ and $\varphi$. We denote the principal ideal generated by a morphism $(a, s, b)$ of $C$ by $\langle a, s, b\rangle$, and observe that $\langle a, s, b\rangle=\left\langle a, s s^{-1}, a\right\rangle=\left\langle b, s^{-1} s, b\right\rangle$. We define the sets $\mathscr{X}_{S}, \mathscr{Y}_{S}$ and $\mathscr{Z}_{S}$ as follows:

(1) $\mathscr{Z}_{S}$ is the set of ideals of $C$;

(2) $\mathscr{X}_{S}=\{\langle a, e, a\rangle: a \in G, e \in E(S)\}$;

(3) $\mathscr{Y}_{S}=\{\langle 1, e, 1\rangle: e \in E(S)\}$.

The set $\mathscr{Z}_{S}$ is partially ordered by inclusion and hence so are its subsets $\mathscr{X}_{S}$ and $\mathscr{Y}_{s}$. Each has least element $I$ where $I=\langle a, 0, a\rangle$ for any $a \in G$. Clearly $\mathscr{Z}_{S}$ is a semilattice under this partial ordering. Notice that by (2) of Proposition 1.6, $\mathscr{Y}_{S}$ is in one-one correspondence with $E(S)$. 
LEMMA 2.1. If $e, f \in E(S)$, then $\langle 1, e, 1\rangle \cap\langle 1, f, 1\rangle=\langle 1, e f, 1\rangle$ and consequently, $\left(\mathscr{Y}_{S}, \cap\right)$ is a semilattice isomorphic to $E(S)$.

Proof. Suppose first that $(a, s, b) \in\langle 1, e, 1\rangle \cap\langle 1, f, 1\rangle$ for some $s \neq 0$. Then, for some $u, v, w, z \in S^{*}$ we have

$$
(a, s, b)=(a, u, 1)(1, e, 1)(1, v, b)=(a, w, 1)(1, f, 1)(1, z, b)
$$

where $u \varphi=a^{-1}=w \varphi, v \varphi=b=z \varphi$, and $u e v=w f z=s \neq 0$. The last equality implies $u^{-1} w \neq 0$ so that $\left(u^{-1} w\right) \varphi=(u \varphi)^{-1}(w \varphi)=1$ giving $u^{-1} w \in E^{*}(S)$. Now $u^{-1} u e v=u^{-1} w f z=f u^{-1}$ wf $z=f u^{-1} u e v=u^{-1} u e f v$ so that $u e v=$ uef $v$. Hence ef $\neq 0$ and $(a, s, b)=(a$, uef $v, b)=(a, u, 1)(1, e f, 1)(1, v, b) \in\langle 1, e f, 1\rangle$.

On the other hand, we see that $(1, e f, 1)=(1, e, 1)(1, f, 1) \in\langle 1, e, 1\rangle \cap\langle 1, f, 1\rangle$, and so the claim is established.

We extend the action of $G$ on $C$ to an action on $\mathscr{Z}_{S}$ by putting $g J=\{g x: x \in J\}$ for every $J \in \mathscr{Z}_{S}$. Note that $g(J \cap K)=g J \cap g K$ for any $g \in G$ and $J, K \in \mathscr{Z}_{S}$ so that we can form the 0 -semidirect product $\mathscr{Z}_{S} *_{0} G$.

If $p, q \in$ Mor $C$ and $p \mathscr{J} q$, then, as noted in [4, Proposition 3.5], $g p \mathscr{J} g q$ for all $g \in G$. It follows that $\mathscr{X}_{S}$ is a $G$-subset of $\mathscr{Z}_{S}$, that is, $\mathscr{X}_{S}$ is closed under the action of $G$. Indeed, if $g, a, b \in G$ and $s \in S$, then $g\langle a, s, b\rangle=\langle g a, s, g b\rangle$. Clearly, $G$ acts on $\mathscr{X}_{S}$ by order automorphisms and $g \mathscr{Y}_{S} \cap \mathscr{Y}_{S} \neq \emptyset$ for any $g \in G$, since $g I=I$.

Since $\langle a, e, a\rangle=a\langle 1, e, 1\rangle$ for every $\langle a, e, a\rangle \in \mathscr{X}_{S}$ we have $\mathscr{X}_{S}=G \mathscr{Y}_{S}$.

LEMMA 2.2. With the above notation, $\left(G, \mathscr{X}_{S}, \mathscr{Y}_{S}\right)$ is a McAlister 0-triple.

Proof. All that remains is to show that $\mathscr{Y}_{S}$ is an order ideal in $\mathscr{X}_{S}$. Suppose that $a \in G$ and $e, f \in E^{*}(S)$ are such that $\langle a, e, a\rangle \subseteq\langle 1, f, 1\rangle$. Then $(a, e, a)=(a, u, 1)(1, f, 1)(1, v, a)$ for some $(a, u, 1),(1, v, a) \in C$. Since $(a, e, a)$ is idempotent, we obtain

$$
(a, e, a)=(a, u f, 1)(1, v u f v u, 1)(1, f v, a)
$$

so that $(a, e, a) \in\langle 1, v u f v u, 1\rangle$. Also,

$$
(1, v u f v u, 1)=(1, v, a)(a, e, a)(a, u, 1)
$$

and so $(1, v u f v u, 1)$ is in $\langle a, e, a\rangle$. It follows that $\langle a, e, a\rangle=\langle 1, v u f v u, 1\rangle$ and consequently, $\langle a, e, a\rangle \in \mathscr{Y}_{S}$. Thus $\mathscr{Y}_{S}$ is an order ideal in $\mathscr{X}_{S}$ as required.

The next lemma, which follows from parts (4) and (6) of Proposition 1.6 will be used repeatedly in what follows. 
LEMMA 2.3. Let $S$ be a strongly $E^{*}$-unitary inverse semigroup and $\varphi$ be an idempotent-pure 0-morphism to a group with zero. If $(a, e, a)$ and $(b, f, b)$ are elements of $C$, (where necessarily $e, f \in E(S)$ ), then $\langle a, e, a\rangle=\langle b, f, b\rangle$ if and only if $e=f=0$ or there is a non-zero element s of $S$ such that $e=s s^{-1}, f=s^{-1} s$, and $a(s \varphi)=b$.

COROLLARY 2.4. With the above notation,

$$
\left\langle 1, s s^{-1}, 1\right\rangle=\left\langle s \varphi, s^{-1} s, s \varphi\right\rangle .
$$

We are now ready to give the main result of this section.

THEOREM 2.5. Let $S$ be an inverse semigroup with zero. Then, the following statements are equivalent:

(1) $S$ is strongly $E^{*}$-unitary;

(2) $S \cong \mathscr{M}_{0}(G, \mathscr{X}, \mathscr{Y})$ for some McAlister 0-triple $(G, \mathscr{X}, \mathscr{Y})$;

(3) $S$ is isomorphic to an inverse subsemigroup containing zero of a 0 -semidirect product $Y *_{0} G$ where $G$ is a group acting by order automorphisms on a semilattice $Y$ with zero;

(4) $S$ is isomorphic to $C_{u} / I_{u}$, where $C$ is an inverse locally idempotent and locally commutative semigroupoid on which a group $G$ acts transitively and freely, $u \in \mathrm{Ob} C$, and $I$ is an ideal of $C$ with $G I \subseteq I$.

PROOF. The implications (2) implies (1), (3) implies (1), and (4) implies (1) are given by Coroliary 1.8, Corollary 1.9, and Proposition 1.5, respectively.

We now prove that (1) implies (2). Since $S$ is a strongly $E^{*}$-unitary inverse semigroup, there is a group $G$ and an idempotent-pure 0 -morphism $\varphi: S \rightarrow G^{0}$. By Lemma $2.2,\left(G, \mathscr{X}_{S}, \mathscr{Y}_{S}\right)$ is a McAlister 0 -triple and so we can form the semigroup $\mathscr{M}_{0}\left(G, \mathscr{X}_{S}, \mathscr{Y}_{S}\right)$. We now show that this semigroup is isomorphic to $S$. Let

$$
\gamma: S \rightarrow \mathscr{M}_{0}\left(G, \mathscr{X}_{S}, \mathscr{Y}_{S}\right)
$$

be defined by $s \gamma=\left(\left\langle 1, s s^{-1}, 1\right\rangle, s \varphi\right)$ if $s \neq 0$, and $0 \gamma=0$. By Corollary 2.4, $(s \varphi)^{-1}\left\langle 1, s s^{-1}, 1\right\rangle=\left\langle 1, s^{-1} s, 1\right\rangle$ so that $(s \varphi)^{-1}\left\langle 1, s s^{-1}, 1\right\rangle$ is a member of $\mathscr{Y}_{S}$ and $s \gamma \in \mathscr{M}_{0}\left(G, \mathscr{X}_{s}, \mathscr{Y}_{s}\right)$ as required.

To see that $\gamma$ is injective, note that if $s \gamma=t \gamma$ and $s \neq 0$, then $t \neq 0, s \varphi=t \varphi$, and

$$
(1, s, s \varphi) \mathscr{J}\left(1, s s^{-1}, 1\right)=\left(1, t t^{-1}, 1\right) \mathscr{J}(1, t, t \varphi),
$$

whence $s=t$ by part (3) of Proposition 1.6.

To show that $\gamma$ is surjective, take any $(\langle 1, e, 1\rangle, g)$ with $e \neq 0$ and $\left\langle g^{-1}, e, g^{-1}\right\rangle=$ $\langle 1, f, 1\rangle \in \mathscr{Y}_{s}$. By Lemma 2.3, there exists $s \in S^{*}$ such that $s \varphi=g, e=s s^{-1}$ and $f=s^{-1} s$. Then $s \gamma=(\langle 1, e, 1\rangle, g)$, as desired. 
To see that $\gamma$ is a morphism, suppose that $s, t \in S^{*}$. Then, taking products in $\mathscr{M}\left(G, \mathscr{X}_{s}, \mathscr{Y}_{s}\right)$ and using Corollary 2.4 ,

$$
\begin{aligned}
s \gamma t \gamma & =\left(\left\langle 1, s s^{-1}, 1\right\rangle, s \varphi\right)\left(\left\langle 1, t t^{-1}, 1,\right\rangle, t \varphi\right) \\
& =\left(\left\langle 1, s s^{-1}, 1\right\rangle \cap s \varphi\left\langle 1, t t^{-1}, 1\right\rangle, s \varphi t \varphi\right) \\
& =\left(s \varphi\left(\left\langle 1, s^{-1} s, 1\right\rangle \cap\left\langle 1, t t^{-1}, 1\right\rangle\right), s \varphi t \varphi\right) .
\end{aligned}
$$

If $s t=0$, then by Lemma 2.1 ,

$$
\left\langle 1, s^{-1} s, 1\right\rangle \cap\left\langle 1, t t^{-1}, 1\right\rangle=\{I\}
$$

so that $s \gamma t \gamma \in\{I\} \times G$, and, viewed as a product in $\mathscr{M}_{0}\left(G, \mathscr{X}_{S}, \mathscr{Y}_{S}\right)$, we have $s \gamma t \gamma=0=(s t) \gamma$. On the other hand, if $s t \neq 0$, then using Lemma 2.1 gives

$$
s \gamma t \gamma=\left(\left\langle s \varphi, s^{-1} s t t^{-1}, s \varphi\right\rangle,(s t) \varphi\right)=\left(\left\langle 1, s t(s t)^{-1}, 1\right\rangle,(s t) \varphi\right)
$$

by parts (4) and (6) of Proposition 1.6 because $1\left(s t t^{-1}\right) \varphi=s \varphi$, st $(s t)^{-1}=$ $s t t^{-1}\left(s t t^{-1}\right)^{-1}$ and $\left(s t t^{-1}\right)^{-1} s t t^{-1}=s^{-1} s t t^{-1}$. Hence $s \gamma t \gamma=(s t) \gamma$ as required.

The proof of (2) implies (3) is accomplished by observing that $\mathscr{M}_{0}\left(G, \mathscr{X}_{S}, \mathscr{Y}_{S}\right)$ is an inverse subsemigroup of $\mathscr{Z}_{S} *_{0} G$ and contains the zero of $\mathscr{Z}_{S} *_{0} G$.

Finally, we must show that (1) implies (4). Again let $\varphi: S \rightarrow G^{0}$ be an idempotentpure 0-morphism from $S$ to a group with zero. As shown in Proposition 1.6, the semigroupoid $C$ constructed from $G, S$ and $\varphi$ is inverse, locally idempotent and locally commutative. Also, $G$ acts transitively and freely on $C$ and $G I \subseteq I$. Let $a \in G$. By Proposition 1.5,

$$
I_{a}=\left\{(p, g) \in C_{a}: p \in I\right\}
$$

is an ideal of $C_{a}$ and we may thus form the Rees quotient $C_{a} / I_{a}$. We show that this is isomorphic to $S$.

Let $\theta: S \rightarrow C_{a} / I_{a}$ be defined by

$$
s \theta= \begin{cases}\left((a, s, a(s \varphi)), a(s \varphi) a^{-1}\right) & \text { if } s \neq 0 \\ I_{a} & \text { if } s=0\end{cases}
$$

Certainly $\theta$ is a bijection.

Let $s, t \in S^{*}$. Then multiplying in $C_{a}$,

$$
\begin{aligned}
s \theta t \theta & =\left((a, s, a(s \varphi)), a(s \varphi) a^{-1}\right)\left((a, t, a(t \varphi)), a(t \varphi) a^{-1}\right) \\
& =\left((a, s t, a(s \varphi)(t \varphi)), a(s \varphi)(t \varphi) a^{-1}\right)
\end{aligned}
$$

Now if $s t=0$, then $s \theta t \theta \in I_{a}$ so that viewed as a product in $C_{a} / I_{a}$,

$$
s \theta t \theta=I_{a}=(s t) \theta
$$


On the other hand, if $s t \neq 0$, then as $\varphi$ is a 0 -morphism, $(s t) \varphi=(s \varphi)(t \varphi)$ and

$$
s \theta t \theta=\left((a, s t, a(s t) \varphi), a(s t) \varphi a^{-1}\right)=(s t) \theta .
$$

Thus $\theta$ is an isomorphism. This completes the proof of the theorem.

\section{Strongly categorical $E^{*}$-unitary inverse semigroups}

We recall from Section 1 that an inverse semigroup with zero is strongly categorical if it is categorical at zero and has the property that the intersection of any two non-zero ideals is non-zero.

PROPOSITION 3.1. Let $S$ be an $E^{*}$-unitary inverse semigroup. If $S$ is strongly categorical, then $S$ is strongly $E^{*}$-unitary.

PROOF. Since every strongly categorical $E^{*}$-unitary inverse semigroup admits an idempotent-pure 0-restricted homomorphism onto a Brandt semigroup [2], the following suffices.

Let $B=\mathscr{M}^{0}(G ; I, I ; \Delta)$ be a Brandt semigroup. Let $H$ be a group in one-one correspondence with $I$; for simplicity, we assume $H=I$. It is easy to verify that the mapping $\varphi: B \rightarrow(G \times H)^{0}$ defined by $(c, g, d) \varphi=\left(g, c d^{-1}\right)$ and $0 \varphi=0$ is an idempotent-pure 0-morphism from $B$ to (indeed, onto) a group with zero, as required.

Not every strongly $E^{*}$-unitary inverse semigroup is strongly categorical. For example, the three element semilattice containing a pair of incomparable elements is strongly $E^{*}$-unitary but not strongly categorical.

In [2] Gomes and Howie give a ' $P$-theorem' for strongly categorical $E^{*}$-unitary inverse semigroups in terms of Brandt semigroups acting by partial order-automorphisms on a partially ordered set. We now describe their construction.

Let $\mathscr{X}$ be a partially ordered set containing a least element 0 . Departing from our usual notation, we regard partial maps on $\mathscr{X}$ as acting on the left of their arguments. A partial (order)-automorphism of $\mathscr{X}$ is an order-isomorphism $\alpha: \mathscr{A} \rightarrow \mathscr{B}$ where $\mathscr{A}$ and $\mathscr{B}$ are subsets of $\mathscr{X}$. Given such an $\alpha$ we denote its domain $\mathscr{A}$ by dom $\alpha$ and its image $\mathscr{B}$ by $\operatorname{im} \alpha$. A partial automorphism $\alpha$ is 0 -restricted if $\operatorname{dom} \alpha$ and im $\alpha$ both contain 0 , and $\alpha(0)=0$. The set $\mathscr{L}_{0}(\mathscr{X})$ of all 0 -restricted partial automorphisms of $\mathscr{X}$ is an inverse subsemigroup of the symmetric inverse semigroup on $\mathscr{X}$. We put

$$
\mathscr{L}_{I}(\mathscr{X})=\left\{\alpha \in \mathscr{L}_{0}(\mathscr{X}): \text { dom } \alpha \text { and im } \alpha \text { are order ideals of } \mathscr{X}\right\}
$$

and note that $\mathscr{L}_{I}(\mathscr{X})$ is an inverse subsemigroup of $\mathscr{L}_{0}(\mathscr{X})$ and that the unique map with domain and image $\{0\}$ is the zero of $\mathscr{L}_{I}(\mathscr{X})$. 
To say that a Brandt semigroup $B$ acts on $\mathscr{X}$ (on the left) by partial orderautomorphisms means that there is a 0 -restricted homomorphism $\varphi: B \rightarrow \mathscr{L}_{I}(\mathscr{X})$. When $B$ acts on $\mathscr{X}$ we write $b * A$ for $(b \varphi)(A)$ and write $\operatorname{dom} b$ and im $b$ for dom $b \varphi$ and $\operatorname{im} b \varphi$ respectively.

For any subset $\mathscr{A}$ of $\mathscr{X}$, we denote the set $\mathscr{A} \backslash\{0\}$ by $\mathscr{A}^{*}$. Let $B$ act on $\mathscr{X}$ and let $\mathscr{Y}$ be a subset of $\mathscr{X}$. The triple $(B, \mathscr{X}, \mathscr{Y})$ is a Brandt triple if conditions (P1) to (P4) are satisfied:

(P1) $\mathscr{Y}$ is a subsemilattice and order ideal of $\mathscr{X}$;

(P2) for all $e \in E^{*}(B)$ and all $P, Q \in \operatorname{dom} e \cap \mathscr{Y}^{*}$, we have $P \wedge Q \neq 0$;

(P3) $B * \mathscr{Y}=\mathscr{X}$;

(P4) $\left(b * \mathscr{Y}^{*}\right) \cap \mathscr{Y}^{*} \neq \emptyset$ for all $b \in B^{*}$.

The reader might note that we have amalgamated two of the conditions in the list given in [2]. Now let

$$
M=\mathscr{M}(B, \mathscr{X}, \mathscr{Y})=\left\{(P, b) \in \mathscr{Y}^{*} \times B^{*}: b^{-1} * P \in \mathscr{Y}^{*}\right\} \cup\{0\}
$$

where $(B, \mathscr{X}, \mathscr{Y})$ is a Brandt triple. We define a multiplication on $M$ by the rule that

$$
(P, b)(Q, c)= \begin{cases}(P \wedge b * Q, b c) & \text { if } b c \neq 0 \\ 0 & \text { otherwise }\end{cases}
$$

and

$$
(P, b) 0=0(P, b)=00=0 .
$$

Proposition 3.2 ([2]). Let $M=\mathscr{M}(B, \mathscr{X}, \mathscr{Y})$ be defined as above. Then $M$ is a strongly categorical $E^{*}$-unitary inverse semigroup.

Note In [2] it is insisted that the action of $B$ must be faithful, that is, $\varphi$ must be one-one, and effective, that is, $\mathscr{X}=\bigcup_{b \in B}$ dom $B$. However, faithfulness is not needed; the weaker condition that $\operatorname{dom} b \neq\{0\}$ for all $b \in B^{*}$ suffices to construct $P$. Moreover, given a strongly categorical $E^{*}$-unitary inverse semigroup $S$, the recipe provided in [2] to construct a Brandt triple does not always yield a faithful action. For a counterexample, let $S$ be any non-trivial group with zero. We also note that the effectiveness of the action follows from (P3).

Our aim in this section is to prove the converse of Proposition 3.2 directly from our version of the $P$-theorem for strongly $E^{*}$-unitary inverse semigroups.

THEOREM 3.3. Let $S$ be a strongly categorical $E^{*}$-unitary inverse semigroup. Then $S$ is isomorphic to $\mathscr{M}(B, \mathscr{X}, \mathscr{Y})$ for some Brandt triple $(B, \mathscr{X}, \mathscr{Y})$. 
Proof. By Proposition 3.1, $S$ is strongly $E^{*}$-unitary. Thus by Theorem $2.5, S$ is isomorphic to $\mathscr{M}_{0}(G, \mathscr{X}, \mathscr{Y})$, for some McAlister 0-triple $(G, \mathscr{X}, \mathscr{Y})$. Put $P_{0}=$ $\mathscr{M}_{0}(G, \mathscr{X}, \mathscr{Y})$ and note that since $P_{0}$ is categorical at zero, so is $\mathscr{Y}$.

Munn [7] has shown that for a strongly categorical inverse semigroup $S$ the relation

$$
\beta=\left\{(a, b) \in S \times S: e a=e b \neq 0 \text { for some } e=e^{2} \text { in } S\right\} \cup\{(0,0)\}
$$

is a 0 -restricted congruence on $S$ such that $S / \beta$ is a Brandt semigroup. Thus $P_{0} / \beta$ is Brandt. We show that $P_{0} / \beta$ acts on $\mathscr{X}$; our Brandt triple will be

$$
\left(P_{0} / \beta,\left(P_{0} / \beta\right) * \mathscr{Y}, \mathscr{Y}\right) .
$$

The principal order ideal of $\mathscr{X}$ generated an element $C$ of $\mathscr{X}$ is denoted by $(C]$. For a non-zero element $(A, g)$ of $P_{0}$, put

$$
\begin{gathered}
\Delta_{(A, g)}=\{0\} \cup\left\{C \in \mathscr{X}^{*}: \text { for all } D \in(g C]^{*}, \text { there is a lower bound of } A\right. \\
\text { and } \left.D \text { in } \mathscr{Y}^{*}\right\}
\end{gathered}
$$

and note that $\Delta_{(A, g)}$ is an order ideal of $\mathscr{X}$.

Let $(A, g),(B, h) \in P_{0}^{*}$ and suppose that $(A, g) \beta(B, h)$. Then $g=h$ and $A \wedge B \neq$ 0 . We claim that $\Delta_{(A, g)}=\Delta_{(B, h)}$.

Let $C \in \Delta_{(A, g)}$ and let $D \in(C]^{*}$. Then there is an element $T$ of $\mathscr{Y}^{*}$ with $T \leqslant A$ and $T \leqslant g D$. In particular, $T \wedge A \neq 0$ and, since $\mathscr{Y}$ is categorical at zero and $A \wedge B \neq 0$, we have $T \wedge B \neq 0$. It follows that $C \in \Delta_{(B . h)}$. A similar argument shows that $\Delta_{(B, h)}$ is contained in $\Delta_{(A, g)}$ and the claim is proved. Thus the order ideal $\Delta_{(A, g)}$ depends only on the $\beta$-class of $(A, g)$ and we can define an action of $P_{0} / \beta$ on $\mathscr{X}$ by setting $\operatorname{dom}(A, g) \beta=\Delta_{(A, g)}$ for any $(A, g) \beta \in\left(P_{0} / \beta\right)^{*}$, and

$$
(A, g) \beta * B=g B
$$

for all $B \in \Delta_{(A, g)}$. Clearly, every element of $\left(P_{0} / \beta\right)^{*}$ acts as a partial order-automorphism.

To show that our definition determines a 0 -restricted homomorphism from $P_{0} / \beta$ into $\mathscr{L}_{l}(\mathscr{X})$ it suffices to prove that

$$
\operatorname{dom}((A, g)(B, h)) \beta=\operatorname{dom}(A, g) \beta \cap((B, h) \beta)^{-1} \operatorname{dom}(A, g) \beta
$$

for all $(A, g) \beta$ and $(B, h) \beta$ in $\left(P_{0} / \beta\right)^{*}$. Thus we must prove that $C \in \Delta_{(A, g)(B, h)}$ if and only if $C \in \Delta_{(B, h)}$ and $h C \in \Delta_{(A, g)}$.

If $C \in \Delta_{(A, g)(B, h)}^{*}$, then $A \wedge g B \neq 0$ and if $D \in(C]^{*}$, then there is a lower bound $E$ of $A \wedge g B$ and $g h D$ in $\mathscr{Y}^{*}$. Thus $g^{-1} E$ is non-zero and is a lower bound of $B$ and $h D$. Since $g^{-1} E \leqslant B$, we also have $g^{-1} E \in \mathscr{Y}^{*}$ and so $C \in \Delta_{(B, h)}$. Now let $F \in(h C]^{*}$. 
Then $h^{-1} F \in(C]^{*}$ so that $A \wedge g B$ and $g h h^{-1} F$ have a lower bound in $\mathscr{Y}^{*}$ and this must also be a lower bound of $A$ and $g F$. Hence $h C \in \Delta_{(A, g)}$.

Conversely, suppose that $C \in \Delta_{(B, h)}^{*}$ and $h C \in \Delta_{(A, g)}$. If $D \in(C]^{*}$, then there is a lower bound $U$ of $B$ and $h D$ in $\mathscr{Y}^{*}$. Since $U \leqslant h C$ there is a lower bound $V$ of $A$ and $g U$ in $\mathscr{Y}^{*}$. Since $U \leqslant B$ we have $V \leqslant g B$ so that $V \leqslant A \wedge g B$. Further, $V \leqslant g U \leqslant g h D$ so that $V$ is a lower bound of $A \wedge g B$ and $g h D$ and consequently, $C \in \Delta_{(A, g)(B, h)}$ as required.

This completes the proof that there is a 0 -restricted homomorphism $\psi$ from $P_{0} / \beta$ to $\mathscr{L}_{1}(\mathscr{X})$. Put $\mathscr{Z}=\left(P_{0} / \beta\right) * \mathscr{Y}$. Then $\mathscr{Y} \subseteq \mathscr{Z}$ and we claim that $\left(P_{0} / \beta, \mathscr{Z}, \mathscr{Y}\right)$ is a Brandt triple.

If $(A, g) \in P_{0}^{*}$, then $g^{-1} A \in \Delta_{(A, g)} \cap \mathscr{Y}^{*}$. Putting $g=1$ gives $A \in \Delta_{(A, 1)}$ so that $A=1 A=(A, 1) \beta A \in \mathscr{Z}$. Hence $g^{-1} A \in \mathscr{Z}$ and so $\Delta_{(A, g)} \cap \mathscr{Z}$, which is an order ideal of $\mathscr{Z}$, is non-zero. It follows that when the action of $P_{0} / \beta$ is restricted to $\mathscr{Z}$ we get a 0 -restricted homomorphism from $P_{0} / \beta$ into $\mathscr{L}_{I}(\mathscr{Z})$, that is, $P_{0} / \beta$ acts on $\mathscr{Z}$ by partial automorphisms.

We now check that conditions (P1) to (P4) hold with $B$ replaced by $P_{0} / \beta$ and $\mathscr{X}$ by $\mathscr{Z}$. That $(\mathrm{P} 1)$ holds is immediate; $(\mathrm{P} 3)$ is routine and $(\mathrm{P} 2)$ follows from the categoricity at zero of $\mathscr{Y}$. For (P4), let $(A, g) \beta \in\left(P_{0} / \beta\right)^{*}$. Then, as above, $g^{-1} A \in \Delta_{(A, g)} \cap \mathscr{Y}^{*}$ and as $\mathscr{Y} \subseteq \mathscr{Z}$, we have that $(A, g) \beta * g^{-1} A$ is defined and equal to $A$.

We have shown that $\left(P_{0} / \beta, \mathscr{Z}, \mathscr{Y}\right)$ is a Brandt triple. To complete the proof of the theorem we must show that $P_{0}$ is isomorphic to $M=\mathscr{M}\left(P_{0} / \beta, \mathscr{Z}, \mathscr{Y}\right)$.

We define $\theta: P_{0} \rightarrow M$ by putting $0 \theta=0$, and $(A, g) \theta=(A,(A, g) \beta)$ for $(A, g) \in P_{0}^{*}$. Now $((A, g) \beta)^{-1}=(A, g)^{-1} \beta=\left(g^{-1} A, g^{-1}\right) \beta$ and, as noted above, $\left(g^{-1}\right)^{-1}\left(g^{-1} A\right)$ is in $\Delta_{\left(g^{-1} A, g^{-1}\right)} \cap \mathscr{Y}^{*}$. Hence $A \in \Delta_{\left(g^{-1} A, g^{-1}\right)}$ and since $(A, g) \in P_{0}^{*}$ we have $((A, g) \beta)^{-1} A=g^{-1} A \in Y^{*}$. Thus $(A, g) \theta \in M$ and $\theta$ is a map.

That $\theta$ is one-one follows from the fact that $(A, g) \beta=(A, h) \beta$ implies $g=h$. To see that $\theta$ is onto, suppose that $(P,(A, g) B) \in M$. Then $P \in \Delta_{\left(g^{-1} A, g^{-1}\right)}$ and $g^{-1} P \in \mathscr{Y}^{*}$ so that there is a lower bound of $g^{-1} A$ and $g^{-1} P$ in $\mathscr{Y}^{*}$. Hence $A \wedge P \neq 0$. Thus $(P, g) \in P_{0}^{*}$ and $(A, g) \beta(P, g)$.

Finally, we show that $\theta$ is a homomorphism. To this end, let $(A, g),(B, h) \in$ $\left(P_{0} / \beta\right)^{*}$. If $A \wedge g B=0$ then certainly $(A, g) \beta(B, h) \beta=0 \beta$ and $((A, g)(B, h)) \theta=$ $0=(A, g) \theta(B, h) \theta$. On the other hand, if $A \wedge g B \neq 0$, then $A \wedge g B$ is the meet of $A$ and $g B$ in $\mathscr{Z}$ and

$$
\begin{aligned}
(A, g) \theta(B, h) \theta & =(A,(A, g) \beta)(B,(B, h) \beta) \\
& =(A \wedge(A, g) \beta * B,(A, g) \beta(B, h) \beta) \\
& =(A \wedge g B,(A \wedge g B, g h) \beta)=(A \wedge g B, g h) \theta \\
& =((A, g)(B, h)) \theta
\end{aligned}
$$

as required. 


\section{References}

[1] J. Almeida, J.-E. Pin and P. Weil, 'Semigroups whose idempotents form a subsemigroup', Math. Proc. Cambridge Philos. Soc. 111 (1992), 241-253.

[2] G. M. S. Gomes and J. M. Howie, 'A $P$-theorem for inverse semigroups with zero', Portugal. Math. 53 (1996), 257-278.

[3] J. M. Howie, Fundamentals of semigroup theory (Oxford University Press, Oxford, 1995).

[4] S. Margolis and J. E. Pin, 'Inverse semigroups and extensions of groups by semilattices', J. Algebra 110 (1987), 277-297.

[5] D. B. McAlister, 'Groups, semilattices and inverse semigroups', Trans. Amer. Math. Soc. 192 (1974), 227-244.

[6] — ' 'Groups, semilattices and inverse semigroups, II', Trans. Amer. Math. Soc. 196 (1974), 351-370.

[7] W. D. Munn, 'Brandt congruences on inverse semigroups', Proc. London Math. Soc. 14 (1964), 154-164.

[8] L. O'Carroll, 'Embedding theorems for proper inverse semigroups', J. Algebra 42 (1976), 26-40.

[9] M. B. Szendrei, 'A generalization of McAlister's $P$-theorem for $E$-unitary regular semigroups', Acta Sci. Math. (Szeged) 51 (1987), 229-249.

Department of Mathematics

Wilfrid Laurier University

Waterloo

Ontario N2L 3C5

Canada

e-mail: sbulman@machl.wlu.ca
Department of Mathematics

University of York

Heslington

York YO10 5DD

UK

e-mail: jbf1@york.ac.uk

e-mail: varg1@york.ac.uk 